\title{
ASPECTOS BIOÉTICOS EN LA TOMA DE DECISIONES PARA LA CALIDAD DE LA ATENCIÓN EN SALUD
}

\author{
Víctor Manuel Rico Jaime*, Octaviano Domínguez Márquez**
}

\begin{abstract}
Resumen: Se realiza un estudio interdisciplinario en bioética, referido al campo de la atención médica y a sus atributos de calidad total, con enfoque a la toma de decisiones, integrando los principales aspectos bioéticos-dignidad, autonomía y responsabilidad- como componentes ineludibles en las decisiones orientadas hacia la mejora continua de la calidad total en el vasto campo de la atención médica.

Es un estudio analítico que plantea la ingente necesidad de correlacionar de manera interdisciplinar las áreas de gestión en los servicios de salud y algunos aspectos de la bioética que transitan velados o poco visibles, con la intención de que sean percibidos objetivamente, incluidos en la planeación y estimados en sus consecuencias a través de indicadores concretos.
\end{abstract}

Palabras clave: decisiones, calidad, autonomía

\section{BIOETHICAL ISSUES IN HEALTH CARE QUALITY DECISSION MAKING}

\begin{abstract}
An interdisciplinary study on bioethics is carried out, regarding health care field and its total quality attributes, with focus in decision making, integrating the main bioethical issues -dignity, autonomy and responsibility-as unavoidable components in decisions geared to continuous total quality improvement in the vast field of medical care.

This is an analytic study which puts forward the enormous need to relate in a interdisciplinary way health care services management and some bioethical issues hidden or with little visibility, with the intention of improving awareness of them objectively and to be included in design and consequences estimated though specific indicators.
\end{abstract}

Key words: decisions, quality, autonomy

\section{ASPECTOS BIOÉTICOS NA TOMADA DE DECISÓES PARA A QUALIDADE DA ATENÇÂO EM SAÚDE}

Resumo: Foi realizado um estudo interdisciplinar em bioética, referido ao campo da atenção médica e a seus atributos de qualidade total, com enfoque na tomada de decisóes, integrando os principais aspectos bioéticos -dignidade, autonomia e responsabilidade- como componentes inquestionáveis das decisóes orientadas para a melhoria continuada da qualidade total no vasto campo da atenção médica.

Num estudo analítico propóe-se a ingente necessidade de correlacionar de maneira interdisciplinar as áreas de gestão nos serviços de saúde e alguns aspectos da bioética que transitam velados ou pouco visíveis, com a intenção de que sejam percebidos objetivamente, incluídos na proposição e estimados em suas consequências através de indicadores concretos.

Palavras-chave: decisões, qualidade, autonomia

** Doctor en Ciencias en Bioética. Presidente de la Academia Nacional Mexicana de Bioética, A.C., México 


\section{Introducción}

De manera resumida, se presenta un estudio referente a un aspecto nuclear en los procesos de la atención médica -la toma de decisiones-, bajo la consideración de relacionar su dinámica con dos componentes ineludibles de la gestión de los procesos de salud-enfermedad: la calidad total en su acepción sencilla, que la entiende como la plena satisfacción del usuario y del oferente de servicios integrados en un binomio inextricable, y los aspectos bioéticos que generalmente se intuyen pero no se develan ni se aprecian de manera objetiva.

La relevancia de la toma de decisiones en la gestión de los procesos de la salud es además doblemente importante cuando se dirige específicamente a conseguir resultados en la calidad total de la atención a la salud. Además de revelar la participación de aspectos fundamentales de la bioética -como la dignidad, la responsabilidad y la autonomía-, que en su aparición nos manifiesta una tendencia humanizante $\mathrm{o}$, en su defecto, un estancamiento en el estilo paternalista, cuando la autonomía es cancelada o la dignidad ofendida.

La toma de decisiones centraliza los procesos de la gestión de la salud desde la planeación, con un sentido estratégico en un nivel medio y, de manera significativa, en la operacionalidad de la atención médica. La toma de decisiones, los paradigmas de la calidad total y los aspectos de la bioética conforman una tríada que es necesario analizar en su trascendencia social, laboral y consecuencial para una óptima atención a la salud.

El ser humano, que ha sido capaz de crear vehículos para la conquista del espacio, a menudo es incapaz de entenderse con su vecino, con sus compañeros de trabajo y, eventualmente, consigo mismo. Se admite que alrededor de $80 \%$ de los problemas serios que aquejan a las organizaciones modernas se refieren al factor humano. Por otra parte, las ciencias que se abocan al estudio y desarrollo de las cosas u objetos materiales se han desarrollado mucho más rápido que referidas a los seres humanos. Sufrimos un desequilibrio desastroso entre tecnología y humanismo.

Rodríguez-Estrada(1) refiere en su obra a Paul Hersey y Kenneth Blanchard, quienes en su libro "La administración y el comportamiento humano" afirman que de cada 30 dólares gastados en investigación en Estados Unidos, 29 son invertidos en objetos materiales o cosas y sólo uno en personas. En los países de América Latina, con sus problemas de marginación, corrupción, desarrollo dependiente y desorganización atávica, la necesidad de actitudes humanistas es aún más apremiante. Latinoamérica, por ejemplo, cuenta con inmensos recursos climatológicos, minerales, forestales, marinos, entre otros, y sin embargo es una región en vías de desarrollo. Es una paradoja viviente; y la clave está en que somos suborganizados, subadministrados y subeducados. Nuestros grandes problemas son las fallas humanas en un marco muy teórico de calidad.

Por fortuna, en los últimos ańos la sociedad latinoamericana ha reaccionado y se ha preocupado por desarrollar en forma racional el capital humano en todos los niveles. Surgen por todas partes programas de capacitación humanista y de calidad, con miras a que cada cual se responsabilice de su futuro, se abra a la colaboración, cuestione sus metas, aclare sus objetivos y los de su institución, comprenda y acepte a la gente y libere sus capacidades, de modo que los equipos aumenten no sólo la productividad sino la calidad en formas creativas. No se trata de aprendizajes convencionales, predeterminados por una escuela o por un maestro, sino del aprendizaje vivo, significativo, que origine cambios interiores reflejados en nuevas conductas hacia la calidad total.

\section{Aspectos conceptuales}

Una gestión efectiva demanda un empleo efectivo de recursos. En efecto, el uso óptimo de los recursos de una organización es el objetivo primordial del proceso de gestión. Todo dirigente -trátese de un comandante de unidad, del director de un hospital, de un alto funcionario gubernamental, de ejecutivos de organizaciones comerciales- es el responsable de la propiedad de otros individuos. La forma de emplear esas propiedades o bienes, orientadas hacia la calidad total, es la responsabilidad fundamental y la base para juzgar su actuación como directivo.

En el campo de la salud, muchas veces los responsables de su gestión se ven imposibilitados de aprovechar y sacar ventajas para su empresa o institución ante cada oportunidad de logro que se les presente. Por ello, la toma de decisiones debe ser un proceso racional(2) de elección para decidir cómo actuar. Es decir, se debe deliberar qué curso de acción es conveniente tomar para emplear los recursos disponibles. Siempre y cuando hayamos seleccionado una sola alternativa, podremos planear -considerando a este proceso como un conjunto de decisiones inteligentes- el desarrollo 
de políticas, estrategias y cursos de acción que más convengan a los objetivos de la calidad total en salud y, de manera significativa, que esas decisiones lleven a condiciones humanizantes.

Todo funcionario, en cada nivel de la organización para la que labora y en cada una de sus funciones, toma decisiones. Éstas no se hacen en el vacío, es decir, cada decisión colectiva o individual afecta las operaciones de todo el sistema organizacional. El punto crucial de la solución de problemas radica en tomar la decisión oportuna. Un ejecutivo que no toma decisiones por miedo, indecisión u otro motivo está destinado al fracaso, porque mientras piensa que es mejor no decidir olvida que no hacer nada es haber ya tomado una decisión: la peor.

El proceso de la toma de decisiones, visto como un elemento nuclear fundamental para optimizar los recursos, requiere incluir una clara intención, tanto para obtener un mejor aprovechamiento de los recursos como para incluir la calidad y definir los aspectos bioéticos que se encuentran formando parte de cada uno de los procedimientos empleados. La conformación bioética debe especificar los valores y corrientes de pensamiento filosófico que deben mantenerse vigentes en la toma de decisiones, no sólo como un respaldo epistemológico y filosófico de fondo, y además velado, sino como una expresión abierta, explícita, susceptible de evaluarse cuantitativa o cualitativamente, e integrada con la factibilidad de la decisión tomada, sin descartar la repercusión jurídica que conlleva.

Incluir los aspectos bioéticos en la toma de decisiones trasciende a la gestión tradicional. Desvela los valores e interpreta las corrientes de pensamiento que recorren el substrato de intencionalidad del estilo de toma de decisiones. Es el entrecruzamiento del manejo de la gestión de recursos técnicos, tecnológicos, financieros con aspectos humanos.

El diccionario de la Real Academia Española(3) define "decisión" como: "determinación, resolución que se toma o que se da a una cosa dudosa". El origen de la palabra nos puede aclarar sus aspectos más esenciales. Decisión, del latín de-cidere, es un término afín a incisión, precisión, preciso, conciso, circuncisión, cisura, cesura, inciso, occiso. Entraña la idea de "cortar y dejar". Supone que un individuo, ante dos o más alternativas que tienen algo de aceptable, toma una y deja las demás.
En tanto, "calidad total" es un concepto que se resume en la obtención de un producto o servicio por un usuario a quien satisfacen sus expectativas(4). En términos de los servicios de salud, debe entenderse como la satisfacción mutua entre el usuario y quien ofrece el servicio, como un binomio inseparable.

Robert Heller afirma que una decisión es un juicio o una elección entre dos o más opciones, y es algo que surge en innumerables situaciones, ya para solucionar un problema o para aplicar una medida(5). Por definición, los directivos han de saber tomar decisiones $\mathrm{y}$, en el tema que nos ocupa, es imprescindible que éstas consideren su consecuencia en calidad y el efecto humanizante a través de la implicación bioética.

\section{Modalidades en las decisiones}

Las decisiones que un directivo debe tomar pueden ser rutinarias, de emergencia o estratégicas. Muchas decisiones son rutinarias, es decir, se repiten las mismas circunstancias y se opta por tomar medidas cuya eficacia ha sido ya comprobada. Cuando se presentan situaciones sin precedentes, las decisiones se toman en ese instante, a medida que se suceden los acontecimientos. Son decisiones de emergencia y pueden ocupar todo el tiempo de un directivo. Tratándose de decisiones enfocadas a la calidad total y al impacto bioético, son decisiones complejas, como en el caso de un paciente con grave condición hemodinámica por pérdida aguda de sangre, que requiere una transfusión de urgencia y el enfermo y sus familiares se niegan por sostener una religión como la de los Testigos de Jehová. En este caso, se plantea la confrontación entre la beneficencia y la autonomía, la calidad y la pérdida de ésta. Además de urgir una decisión, ¿qué es lo correcto desde el punto de vista bioético? La decisión administrativa pasa a segundo término, la sangre se encuentra disponible y su aplicación en términos de calidad, en cuanto a la eficiencia, está indicado.

La forma más relevante de tomar decisiones está relacionada con las decisiones estratégicas: la tarea más importante de un directivo consiste, de manera consensuada con su equipo de trabajo, en decidir metas y objetivos y convertirlos en planes concretos o en decisiones secundarias o programas de acción. Las decisiones que inciden directamente y a mediano y largo plazo requieren de un gran conocimiento y experiencia, así como de una visión contextual y de futuro. El sentido globalizador de las decisiones estra- 
tégicas va más allá de una decisión parcial o episódica; pretenden ser totalistas y abarcar el área y el contexto, principalmente, el impacto global respecto de la cobertura de responsabilidad, es decir, las consecuencias mediatas e inmediatas.

El acto de decidir implica un ejercicio de conciencia sobre las finalidades que se pretende alcanzar a través de la decisión. Si algo hay en la vida humana que, por su misma naturaleza, pide un alto grado de conciencia es la toma de decisiones.

\section{Decisiones, calidad total y bioética}

La toma de decisiones bajo la perspectiva de la calidad total y la bioética significa que no puede haber calidad total sin que haya implicados valores o principios fundamentales; velados o sin etiqueta, pero presentes. En el campo de la salud, las actividades naturales del área propician que este binomio se presente con o sin la intencionalidad requerida. Basta el énfasis de algún proceso diseñado para la calidad total o para la satisfacción de un valor para que ambos concurran en mayor o menor grado. Lo trascendente es que tanto la calidad total como los elementos bioéticos pueden y deben objetivarse de manera cuantitativa, con indicadores elaborados para ese efecto.

Desde las postulaciones de Juran(6), respecto de la calidad total como un nivel de desarrollo mayor de la productividad, y de los paradigmas de sistematización e inclusión de esquemas estadísticos indispensables de Ishikawa(7), hasta los preceptos de Deming(8), Kobayashi(9), Crosby(10) y otros, la concretización de la calidad total en logros evaluables abrió el camino para que los aspectos bioéticos fueran planteados de igual manera, a través de indicadores formalmente validados.

Los indicadores más conocidos de carácter bioético se relacionan con la dignidad en la interpretación de tiempo de espera, tanto para recibir atención como en la instalación de un tratamiento o en la realización de un procedimiento; de trato digno en la atención personalizada, en el respeto a la aceptación de un estudio o de un tratamiento. Otros indicadores de tipo bioético están sustentados, por ejemplo, en la responsabilidad y en una gama muy amplia de indicadores, desde el cumplimiento de una cita hasta la atención con resultados eficientes y el cuidado de apego a los pacientes. Además, este último indicador se correlaciona con la responsabilidad del paciente y de sus familiares en el cumplimiento de los deberes de autocuidado, en el vasto campo de la prevención y el fomento de la salud y en la adherencia terapéutica.

Apenas los países en vías de desarrollo empiezan a controlar de las afecciones infecto-contagiosas cuando, en esa transición epidemiológica, ya tienen encima las enfermedades crónico-degenerativas(11). De tal manera que las altas tasas de daño y mortalidad de los padecimientos -obesidad, diabetes tipo 2, infarto del miocardio y neoplasias principalmente- podrían ser detenidas con la responsabilidad por parte del enfermo y sus familiares, con base en el cambio de estilo de vida.

Volviendo al tema que nos ocupa, Rodríguez-Estrada(1) describe en su obra "Manejo de problemas y toma de decisiones" la siguiente tipología:

\section{A. Según el sujeto que decide}

Decisiones individuales y decisiones grupales (tomadas por un grupo a través de una discusión).

Dentro de las primeras caben algunas decisiones famosas que han afectado a muchos. Individual fue, por ejemplo, la decisión de Hitler de encender una chispa que se convertiría en la conflagración bélica más devastadora de la historia. Una diferencia apreciable entre ambas es que en las individuales el proceso de la deliberación no existe, en tanto que en las grupales se hace transparente. En términos de la calidad total y de la bioética las decisiones unipersonales no son apreciadas y revelan la persistencia de un estilo sociobiologista, impositivo, autócrata, que rompe con los principios de ambas áreas. La cancelación de la multiparticipación y de la consideración humanizada del acto de decidir no puede coincidir con los actos atávicos de la soledad del mando.

En este punto resalta la preponderancia de la deliberación, del trabajo en equipo, de la consideración prioritaria de considerar al enfermo y a sus familiares como parte activa y no pasiva de ese equipo de trabajo. Abandonar una beneficencia paternalista por una beneficencia que promueva la autonomía como un gran valor.

De tal manera que el consentimiento bajo información, uno de los indicadores universalmente aceptados, tiene en sí la carga del reconocimiento de la autonomía, es de- 
cir, la expresión de la libertad del paciente, no sólo para los procesos de investigación, sino para la aceptación o no de las intervenciones de estudio o de tratamiento. Este indicador puede ser tan sencillo como registrar si fue aplicado o no, o tan complejo como determinar si fue entendido o no por el enfermo, y si además se cumplieron los términos del acuerdo.

La tecnología moderna ha creado gran cantidad de medios capaces de reunir información, pero que en ningún caso pueden reemplazar a una persona en la toma de decisiones. Los sistemas de computación, investigación operativa, modelos matemáticos, entre otros, se consideran sólo como instrumentos para analizar los problemas. El que decide no debe transferir la responsabilidad de la toma de decisiones ni a las máquinas ni a los especialistas en dichas técnicas, sino obtener de ellos el respaldo en datos y referencias para la clasificación del problema y su análisis correspondiente.

\section{B. Según las repercusiones que originan}

Decisiones sobre uno mismo: cuando el médico, enfermera o el propio paciente deciden sobre sí mismos alguna actividad que aparentemente sólo a ellos interesa o afecta. Las decisiones individuales no pueden ser una abstracción de la realidad, mucho menos si están relacionadas con aspectos claros que inciden en la calidad total y en la atención humanista, por ser el individuo parte de un grupo. A pesar de que muchas decisiones son necesariamente tomadas de manera unipersonal, por considerar que sus repercusiones no trascienden o afectan a otros, deben ser valoradas cuidadosamente.

Decisiones sobre otros: en los casos en que los directivos o el personal de salud toman decisiones generalmente sin la participación de aquellos a los que afecta la decisión, las repercusiones son importantes. La calidad total puede verse severamente afectada, dado que los individuos, al no ser tomados en cuenta, hacen caso omiso de su responsabilidad. Este es uno de los principales problemas que enfrentan los comités de atención asistencial cuando sus resoluciones fueron tomadas sin la participación de los pacientes y familiares, así como también del personal de salud involucrado en el proceso.

\section{Según el nivel jerárquico}

Este punto de vista está estrechamente relacionado con el anterior. Podemos observar tres tipos de decisiones según el nivel jerárquico: operativas, tácticas y estratégicas. Refiriéndonos al campo de la salud, cada una de ellas es tan importante como las restantes.

Decisiones operativas son las que competen a los médicos, no sólo por su actuar profesional en cuanto a los aspectos preventivos y curativos, sino a sus actividades comunes y rutinarias que generalmente no son apreciadas en su trascendencia. El segundo gran costo en el presupuesto de las instituciones de salud es el gasto en medicamentos, de ahí que la racionalidad de la prescripción médica es sumamente importante, además de sus efectos en la enfermedad para la cual fue indicada. También tienen un costo importante las decisiones en exámenes de laboratorio, imagenología, medicina nuclear y otros procedimientos de carácter invasivo. Pero aquel en el que radica buena parte de la salud financiera de las instituciones es el otorgamiento de licencias o incapacidades, temporales o permanentes, totales o parciales. En efecto, se considera que sobre el médico general y el de especialidad gravita un fuerte componente económico, dependiente de sus decisiones.

Las decisiones tácticas se manejan en el nivel de gerentes, jefes de sección o de departamento. Este grupo, considerado como el mando medio de las organizaciones, es responsable de la interpretación de las decisiones estratégicas y de las políticas, y de la conducción de los grupos en ambientes de armonía y optimismo hacia el logro de la calidad total. Los mandos medios son los que deben identificar el avance correcto de las fases de los procesos con toda oportunidad para conseguir su corrección. La capacitación más efectiva y valiosa está precisamente ejecutada por los mandos medios en la observancia de los actos operativos. Cuando este nivel decisorio falla, el nivel operativo se vuelve caótico y los resultados en la calidad total y en los componentes bioéticos se revelan en quejas, inconformidades y errores graves. Las demandas no se hacen esperar.

Las decisiones estratégicas competen a los individuos con responsabilidades en alta dirección: secretarios de Estado o ministros, directores generales, entre otros. Son quienes, con su equipo de trabajo, fijan los objetivos y políticas generales de las instituciones. Asimismo, evalúan periódicamente los resultados y efectos de la calidad total, la productividad y los aspectos bioéticos, estos últimos apreciados de manera generalmente indirecta. 
La tipología puede ampliarse. Hay decisiones prudentes e imprudentes, oportunas o inoportunas, intelectuales y sentimentales; decisiones para librarse de un problema o para procurarse un beneficio de ejecución inmediata o diferida; genuinas, que expresarán la voluntad de las personas y grupos, y manipuladas, verdaderas extorsiones y abusos de la buena fe o de la torpeza de la gente.
Finalmente, las decisiones son un proceso imprescindible, delicado, complejo y fundamental en la consecución de la calidad total y de la objetivación de los aspectos bioéticos en el campo de la salud, que no sólo compete a los directivos sino a toda la organización, incluyendo al paciente y a sus familiares.

\section{Referencias}

1. Rodríguez-Estrada M. Manejo de problemas y toma de decisiones. México: El Manual Moderno; 1988.

2. Koontz H, Weilhrich H. Administración. Una perspectiva global. México; McGraw-Hill Interamericana; 2008: 158.

3. Real Academia de la Lengua. Diccionario de la Lengua Española. Madrid: Espasa-Calpe; 2001: 731-733.

4. Feigenbaum AV. Control total de la calidad. México: Compañía Editorial Continental; 1992: 74-75.

5. Heller R. La toma de decisiones. Barcelona: Grijalbo-Mondadori; 1998.

6. Jurán JM. Jurán y el liderazgo para la calidad. Madrid: Díaz de Santos; 1990.

7. Ishikawa K. ¿Qué es el control total de calidad? México: Norma; 1994.

8. Gitlow HS, Gitlow SJ. Cómo mejorar la calidad y la productividad con el método Deming. Colombia: Norma; 1990.

9. Kobayashi S. Administración creativa. México: Editora Técnica; 1982.

10. Crosby P. Calidad sin lágrimas. México: Compañía Editorial Continental; 1989.

11. Tapia CR. El manual de salud pública. Tomo I. México: Intersistemas; 2004: 137-139.

Recibido: 23 de junio de 2010

Aceptado: 19 de julio de 2010 